EPSC Abstracts

Vol. 14, EPSC2020-1000, 2020, updated on 18 Nov 2020

https://doi.org/10.5194/epsc2020-1000

Europlanet Science Congress 2020

(C) Author(s) 2020. This work is distributed under

the Creative Commons Attribution 4.0 License.

\title{
Jupiter's aurora liveliness during solar minimum
}

Benjamin Palmaerts ${ }^{1}$, Denis Grodent ${ }^{1}$, Bertrand Bonfond ${ }^{1}$, Zhonghua Yao ${ }^{1,2}$, Ruilong Guo ${ }^{1}$, Maïté Dumont $^{1}$, Jean-Claude Gérard ${ }^{1}$, and Kamolporn Haewsantati ${ }^{1,3}$

${ }^{1}$ LPAP, University of Liege, Liege, Belgium (b.palmaerts@uliege.be)

${ }^{2}$ Key Laboratory of Earth and Planetary Physics, Chinese Academy of Sciences, Beijing, China

${ }^{3}$ Department of Physics and Materials Science, Chiang Mai University, Chiang Mai, Thailand

Although studied for many years, the powerful ultraviolet auroral emissions at Jupiter still contain many mysteries. Even well-established theories explaining the Jovian main auroras are now questioned in the light of observations by the Juno spacecraft currently orbiting the giant planet. Jupiter's aurora is known to respond to changes in solar wind on one hand and to processes occurring inside the magnetosphere on the other hand. However, many changes regularly observed in the aurora could not yet be categorized as solar wind-driven or as internally-driven dynamics. An observing campaign of the Jovian aurora with the Hubble Space Telescope (HST) has been performed between February and September 2019 (HST GO-15638), including approximately 10 visits around each of the perijoves of Juno's orbits 18 to 22. During this time, the solar activity was minimal, giving the opportunity to investigate auroral dynamics mainly controlled by internal processes. The main emission often appeared dim and diffuse (see the example on Figure 1), in particular on the dawn side where a narrow arc is generally found. In contrast, emissions poleward of the main emission were very dynamic, exhibiting some periodic brightening and intensities occasionally increasing tenfold over a few minutes (like in the middle panel of Figure 1). Many other interesting features are observed, such as dawn storms, duplication of the main emission, fresh and old injection signatures and transpolar arcs. All of these emissions are investigated by combining HST high temporal and spatial resolution images with in situ data simultaneously collected by Juno in Jupiter's magnetosphere. Additionally, some HST visits have been scheduled while Juno-UV Spectrograph was observing the opposite hemisphere at the same time, enabling the tracking of conjugate auroral features in both hemispheres simultaneously.

Figure 1: Sequence of polar projections of HST images of the northern aurora at Jupiter, taken on September 10, 2019. 


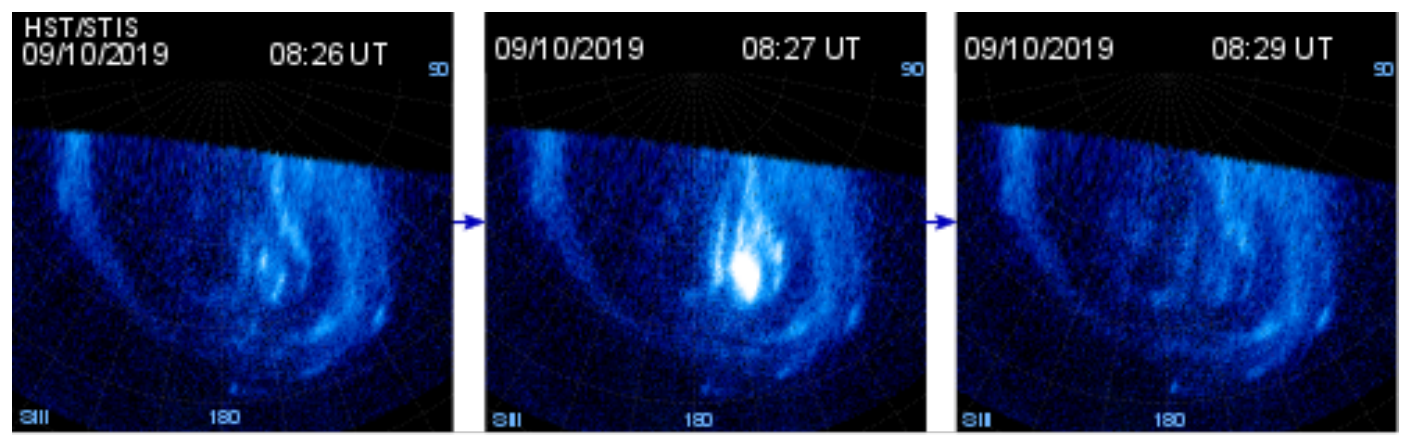

